\title{
Philosophiques
}

\section{Maurice Gagnon et Daniel Hébert, 2000, En quête de science. Introduction à l'épistémologie, Montréal, Fides.}

\section{André Lacroix}

Volume 28, numéro 1, printemps 2001

La nature des normes

URI : https://id.erudit.org/iderudit/004939ar

DOI : https://doi.org/10.7202/004939ar

Aller au sommaire du numéro

Éditeur(s)

Société de philosophie du Québec

ISSN

0316-2923 (imprimé)

1492-1391 (numérique)

Découvrir la revue

Citer ce compte rendu

Lacroix, A. (2001). Compte rendu de [Maurice Gagnon et Daniel Hébert, 2000,

En quête de science. Introduction à l'épistémologie, Montréal, Fides.]

Philosophiques, 28(1), 236-237. https://doi.org/10.7202/004939ar d'utilisation que vous pouvez consulter en ligne.

https://apropos.erudit.org/fr/usagers/politique-dutilisation/ 


\section{Philosophiques / Printemps 2001}

\section{Maurice Gagnon et Daniel Hébert, 2000, En quête de science. Introduction à l'épistémologie, Montréal, Fides.}

Préfacé par Jean-François Malherbe, En quête de science est un livre de synthèse. Ses auteurs, les professeurs Gagnon et Hébert enseignent la philosophie à Sherbrooke depuis de nombreuses années. Le premier mène des recherches en épistémologie à l'Université de Sherbrooke depuis plus de trente ans tandis que le second enseigne la philosophie au Cégep de Sherbrooke depuis quelques années. Dans leur livre, ces deux philosophes ont voulu présenter et discuter les principaux concepts utilisés en épistémologie. Pour ce faire, ils ont retenu une présentation conceptuelle plutôt que thématique afin d'amener le profane à se familiariser avec les concepts élaborés au fil des siècles par les épistémologues. Cela, sans entrer dans le détail de toutes les querelles et polémiques parmi les plus pointues ayant cours dans le champ de l'épistémologie. Là n'est pas l'ambition des auteurs.

L'ouvrage est divisé en 9 chapitres. Dans les premiers chapitres, les auteurs ont d'abord souhaité situer le lecteur en discutant de la perception et de la conceptualisation. Choix éclairant à plus d'un titre qui a le mérite de situer l'ouvrage sur le terrain de la psychologie de la connaissance afin d'amener le lecteur à mieux mesurer l'importance de l'épistémologie. Les auteurs présentent en effet cette discipline sous l'angle de notre appréhension du monde : comment concevons-nous le monde qui nous entoure et comment le comprenons-nous ? Plutôt que de discuter abstraitement des différentes théories épistémologiques et d'en présenter les points forts et les points faibles, Gagnon et Hébert ont en effet préféré aborder la réflexion épistémologique sous l'angle somme toute assez classique de la connaissance entendue comme épistémé, comme perception. Et sur ce point, les deux premiers chapitres consacrés à la discussion de la perception et de la conceptualisation de la connaissance ont le mérite de placer l'être humain au centre des processus cognitifs.

Ce choix fait, et l'humain mis face à l'appréhension du savoir, les auteurs se sont ensuite arrêtés à présenter la structure de la connaissance afin de montrer comment notre savoir se constitue. Pour rendre la présentation la plus éclairante possible, ils ont retenu la discipline la mieux structurée, celle qui sert à tout le moins de paradigme à la plupart des discussions en épistémologie, soit la physique, pour discuter les concepts et fournir des exemples. Les chapitres 2 à 6 y sont presque totalement consacrés alors que les notions d'hypothèse, d'induction, de lois et de mesure pour ne nommer que celles-là y sont successivement traitées.

Le point de départ de la connaissance situé, soit la place que l'être humain occupe dans la construction du savoir, et la structure de ce savoir fixé, il restait encore à présenter les fondements de ce dernier, son évolution et les principaux problèmes actuellement rencontrés par les sciences du comportement, soit le domaine le moins structuré des sciences, puisque le plus jeune sans aucun doute. Ce sont les chapitres 7,8 et 9 qui y sont consacrés.

Dans le chapitre 7, plutôt que de vouloir traiter tous les concepts servant à fonder la connaissance scientifique, les auteurs ont judicieusement préféré s'arrêter aux principaux concepts, de manière à rendre la présentation plus aisée, quitte à inviter le lecteur à consulter d'autres ouvrages pour approfondir la réflexion. C'est ainsi qu'ils ont choisi de présenter les notions de vérité, de cause, de déterminisme et de hasard, toutes des notions qui sont au cœur de la réflexion épistémologique classique. Exemples à l'appui, chacune des notions est expliquée avant d'être restitués à l'intérieur des 
principales querelles théoriques. Un tel mode d'exposition permet sans nul doute une consultation rapide et une entrée en la matière des plus réussies.

Dans le chapitre 8 enfin, c'est le débat Kuhn, Popper, Lakatos qui occupe Gagnon et Hébert : comment les théories scientifiques évoluent-elles, se modifientelles et se construisent-elles ? Comment, en d'autres termes le savoir se constitue-t-il de manière plus ou moins objective ? Débat maintenant classique, mais qui ne peut néanmoins être apprécié à sa juste valeur que dans la mesure où le néophyte a d'abord été familiarisé avec les concepts épistémologiques de base. D'où la place faite à ce débat au chapitre 8 alors que le lecteur a déjà assimilé les notions de base et est maintenant en mesure de saisir les importants enjeux du débat.

Enfin, en se penchant sur les principaux problèmes rencontrés par les sciences du comportement, les auteurs bouclent la boucle puisqu'ils reviennent à leur point de départ : comment l'être humain interagit-il avec son milieu ? Les auteurs reprennent cette première question qui les a amené à dresser la table de l'épistémologie contemporaine pour relancer le débat et ouvrir sur ce qui fait actuellement problème en épistémologie : la place de l'humain dans la constitution du savoir. Et si ce n'est plus conceptuellement que cette place se discute, c'est sans nul doute au niveau des comportements humains et de la compréhension que nous pouvons en avoir. Comment en effet transposer le cadre des sciences physiques dans ces sciences ? Et si une telle démarche n'est pas possible comme la plupart des chercheurs le reconnaissent maintenant, cela nous contraint-il à revoir notre conception univoque du savoir et de la science ?

Comme tous les spécialistes en conviendront, ces questions sont au centre des débats en épistémologie et choisir de les présenter à la fin d'un ouvrage d'introduction devient ainsi des plus intéressants. Ouvrage de synthèse et ouvrage critique, le livre de Gagnon et Hébert constitue une fort intéressante entrée en la matière pour celui et celle qui souhaiterait s'initier aux grands débats menés en épistémologie. Peut-être que certains spécialistes reprocheraient aux auteurs d'avoir négligé tous les développements entourant les sciences du vivant et la biologie, lesquelles furent au cœur de stimulantes discussions, mais de tels reproches ne sauraient nous faire oublier l'excellente présentation et l'intéressante démarche pédagogique retenue par les auteurs. En fixant les choix théoriques et conceptuels qui colorent l'ouvrage, les auteurs ont opté pour une présentation classique de la matière. Et sur ce point, nous ne saurions guère leur faire de reproches. Enfin, une bibliographie plus consistante aurait sans doute permis au néophyte de se familiariser davantage avec les débats ayant actuellement cours en épistémologie, et lui aurait permis de dépasser les grands classiques qui figurent par ailleurs en bonne place dans la bibliographie.

Mais par-delà ces deux petites réserves qui ont d'ailleurs davantage à voir avec la forme de la présentation retenue qu'avec le fond, nous ne saurions trop recommander la lecture de ce livre d'introduction. D'autant plus que le livre de Gagnon et Hébert est bien présenté et bien documenté de sorte qu'il constitue une fort intéressante introduction à l'épistémologie qui est présentée comme étant la connaissance de la connaissance. Et sur ce plan, les auteurs nous rappellent bien jusqu'à quel point la connaissance reste fragile, reste affaire mouvante qui change au fil du temps.

ANDRÉ LACROIX 